\title{
Optimal Biped Walking with a Complete Dynamical Model
}

\author{
Michael Hardt ${ }^{1}$ \\ mhardt@ece.ucsd.edu \\ Kenneth Kreutz-Delgado \\ kreutz@ece.ucsd.edu \\ Department of Electrical \& Computer Engineering \\ University of California, San Diego \\ La Jolla, CA 92093-0407
}

\author{
J. William Helton ${ }^{1}$ \\ helton@osiris.ucsd.edu \\ Department of Mathematics \\ University of California, San Diego \\ La Jolla, CA 92093-0112
}

\begin{abstract}
We solve the problem of generating symmetric, periodic minimum energy gaits for a 5-link biped robot moving in the sagittal plane of forward motion. We seek to approximate natural walking motion through the minimization of actuation energy. The model we use has considerably more structure than those previously studied. This forces us to a fully nonlinear minimum energy path planning problem on a 14-dimensional state space. Also a large number of constraints must be considered, including contact and collision effects. Our solution required development of various symbolic, dynamical algorithms relating to multibody systems and use of powerful numerical optimal control software. Solving the minimum energy walking problem including saturation and algebraic constraints amounts to solving a Hamilton-Jacobi-Bellman type equation along the optimal path. We use the path planning software DIRCOL which can handle the many constraints, as well as the high degree of nonlinearity and high dimensionality. A newly available version of this software provided a substantial decrease in computing time required for generating solutions. We discuss numerical optimization and other modeling issues in this paper.
\end{abstract}

\section{Introduction}

Modeling and understanding the seemingly simple process of human walking remains as one of the more difficult research problems in multibody systems and robotics due to its complexity and high dimension. There are many variations of walking in humans, though we will concern ourselves only with the periodic motion associated with moving at a constant average speed on a flat surface. Due to the complexity of the problem, compromising simplifying modeling assumptions were often made in previous work to make it more tractable. Even a simple 5-link biped robot with all rotational joints and full motion degrees of freedom will have a 14 dimensional state space when represented with respect to generalized coordinates. One also encounters a differential-algebraic system when contact constraints of the feet with the ground are considered.

\footnotetext{
${ }^{1}$ Partially supported by AFOSR, NSF, \& Ford Motor Co.
}

A very thorough investigation into minimum energy walking with numerical methods was undertaken early on in [1] using a highly simplified model which resulted in a 2-link manipulator. The idea of searching for a passive walking motion which can approximate better the minimum energy motion witnessed in humans was also expressed in the work of McGeer [8] and later with Goswami et al. [4]. The minimum energy path is desirable for it exhibits stabilizing, attractive properties. Our experiments have shown that many walking trajectories, naively chosen to approximate walking motion, can require a huge increase in energy over that of the optimally calculated minimum energy motion. Other recent work also investigating minimum energy motion with simplified models may be found in [10]. Very interesting walking machines were constructed by McGeer [8] and also by Kajita [7]. Kajita modeled the biped dynamics as an inverted pendulum with point masses whose simplicity forced an interesting and unusual construction of the biped.

To properly model walking, one should consider explicitly both phases of walking. The first phase has one foot in contact, while in the shorter second phase both feet are in contact with the ground. The difficulty with this perspective, however, is that one is faced with a differential-algebraic system with a varying number of algebraic constraints. The collision of the foot with the ground results in jump conditions on the velocities while there also exists saturation constraints on the state variables and the actuators. In this paper, we explain how our numerical approach is able to produce solutions which satisfy all of these constraints.

The first step towards reducing the complexity of the problem was made in the actual dynamical modeling of the system using recently developed recursive, symbolic algorithms [5]. Another important step was the creation of a reduced dynamics algorithm for evaluating the unconstrained reduced-dimensional dynamics of the biped which account for the contact constraints [6]. This makes it possible to integrate in time the reduced system rather than the full differential-algebraic dynamical system. Finally, powerful numerical optimal control software (DIRCOL) has been developed [11, 12] which can handle control problems of high dimemsion 
with many forms of constraints. We present here numerical results demonstrating the use of DIRCOL.

In the discussion of our numerical experiments, we present several different model variations. We investigate both the introduction of ankle torques at the point of contact of the legs with the ground as well as the underactuated case when these torques are not added. An additional impulsive liftoff force is added when the swing leg lifts off of the ground thereby creating a smoother motion and simulating better the human's use of the foot to create a liftoff force. Other parameters which are varied are the biped's step length, the time of one step, and the proportion of time corresponding to the contact phase. We also discuss the effect of these parameters on the system energy. Preliminary results for the solution of this problem were first presented in [6].

\subsection{Biped Model}

\section{Model and Dynamics}

Many of the essential characteristics of the human walking motion may be captured with a 5 -link planar biped walking in the two-dimensional sagittal plane, the vertical plane bisecting the front of the biped. The model contains two links for each leg plus a large, massive torso, which also functions as the base of the tree-structured multibody system. Though the motion is constrained to the 2-dimensional vertical sagittal plane, in our experiments we model the links with a 3dimensional elliptical shape and a uniform distribution of mass. The physical data corresponding to the model used in our experiments can be found in Table 1 .

Table 1: Biped Model Physical Data

\begin{tabular}{lrrr} 
Link & Mass & Length & Radius \\
\hline Torso & $20 \mathrm{~kg}$ & $0.72 \mathrm{~m}$ & $0.12 \mathrm{~m}$ \\
Upper Leg & $7 \mathrm{~kg}$ & $0.50 \mathrm{~m}$ & $0.07 \mathrm{~m}$ \\
Lower Leg & $4 \mathrm{~kg}$ & $0.50 \mathrm{~m}$ & $0.05 \mathrm{~m}$
\end{tabular}

Though feet are not included in our biped model, much of their influence may be modeled in ways which do not increase the dimension of the system. From the control perspective, two of the main contributions of the feet, when not expressly considering friction, are the introduction of ankle torques and the liftoff force produced as the heel comes off of the ground. It is possible to include ankle torques in the model by treating these as external forces influencing the tips of each leg at the points of contact. Rather than modeling a liftoff force which lasts the entire duration of the double contact phase, as is normally the case with the foot, we model the liftoff force as an instantaneous impulsive force occurring at the moment of liftoff. This last technique has certain numerical advantages though it cannot completely reproduce the effect of the foot as will be shown in the reports of our numerical experiments.

There are a total of 14 states, 6 control variables, and 1 control parameter in our control problem if an impulse liftoff force is modeled.
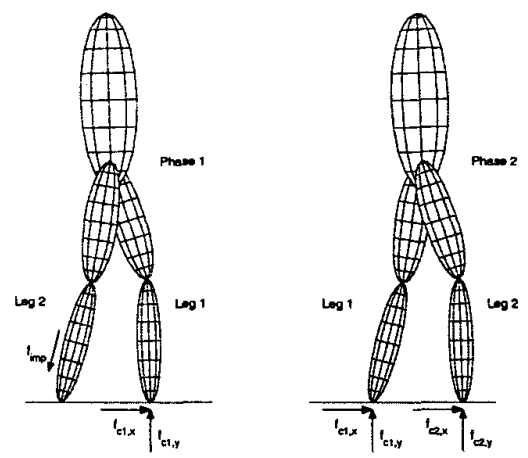

Fig 1: At the beginning of phase 1 an impulse force $f_{i m p}$ can propel the body forward. During phase 2, both legs experience contact forces.

$x_{1}-x_{3}$ torso orientation and pos. in the vert. plane

$x_{4}-x_{6}$ torso angular and linear velocity

$x_{7}, x_{8} \quad$ angle position and velocity of leg 1 hip

$x_{9}, x_{10}$ angle position and velocity of leg 1 knee

$x_{11}, x_{12}$ angle position and velocity of leg 2 hip

$x_{13}, x_{14}$ angle position and velocity of leg 2 knee

$u_{1}, u_{2}$ applied torque at leg 1 hip and knee

$u_{3}, u_{4}$ applied torque at leg 2 hip and knee

$u_{5}, u_{6}$ applied torque at leg $1 \& 2$ ankles

2.2 Recursive, Symbolic Dynamical Algorithms The state equations of the biped walker are those of a multibody system experiencing contact forces,

$$
\ddot{\theta}=\mathcal{M}^{-1}\left(u+J_{c}^{T} f_{c}-\mathcal{C}-\mathcal{G}\right) .
$$

In equation (1), $\mathcal{M}$ is the square, positive-definite mass-inertia matrix, $\mathcal{C}$ is the vector of Coriolis and centrifugal forces, $\mathcal{G}$ is a vector of gravitational forces, $u$ are the applied torques at the links, $J_{c}$ is the constraint Jacobian, and $f_{c}$ is the constraint force.

Several different approaches to recursive, symbolic multibody algorithms were studied, compared, and represented in a unifying formalism in [5]. This work also included the extension of several algorithms to multiple degree of freedom joints and tree-structured systems. The approach is based on decomposing the dynamical quantities into physical, matrix operators. The various link operations are stacked into larger, matrix operators which, in turn, provide a very clean notation which can easily be manipulated for estimation and control design purposes. For high dimensions, recursive, symbolic dynamical models are more efficient for calculating the forward dynamics than other nonrecursive procedures which require constructing and inverting the entire mass-inertia matrix, $\mathcal{M}$.

\subsection{Contact and Reduced Dynamics}

Special recursive algorithms have also been developed to determine the contact forces experienced by the biped under contact and the resulting generalized accelerations $\ddot{\theta}$ which are produced. See [6] and the references found therein. An important component of our 
dynamical modeling which also contributed to our ability to solve this problem was the development of a Reduced Dynamics Algorithm, also described in [6], which makes use of the Contact Algorithm. Because of the contact constraints, we are faced with a differentialalgebraic system. Two courses of actions are possible when it is necessary to integrate the dynamics, one being the use of specially tailored integration routines which often require the partial derivatives of the various contact constraints. The preferable approach, however, is to use a reduced unconstrained set of dynamics which evolve on the constraint manifold. Then it is possible to use standard integration procedures. This latter approach is the one we take.

In the first phase of the biped motion, where one leg is swinging, the contact constraints reduce the total degrees of freedom from 7 to 5 . Thus, using the Reduced Dynamics Algorithm, an unconstrained 10-dimensional state space can represent the system during this period instead of the full 14 dimensions for the completely free system. The remaining 4 states and their time derivatives can be determined from the 10 independent states and their time derivatives. Similarly in the shorter second phase, when both feet are in contact with the ground, contact constraints allows us to work with a system with only a 6-dimensional state space.

\subsection{Collision and Impulsive Forces}

Closely related to the Contact Algorithm is the Collision Algorithm. This algorithm calculates the discontinuous jump in generalized velocities resulting from inelastic collisions of the multibody system with its environment. We model the collision of the swing leg as it makes contact with the ground in this manner. The recursive algorithm determines the impulsive force experienced by the system which propagates throughout all the links of the multibody system.

In several of our experiments, we introduce an impulsive force along the axis of the leg about to lift off of the ground thereby aiding the biped in maintaining its forward momentum. The Collision Algorithm can also be used to determine the resulting jump in the generalized velocities. The magnitude of the impulsive force becomes an additional control parameter which must be included into the energy criteria to be discussed Section 3. Figure 1 displays both the influence of the contact forces to the biped model and the introduction of an impulsive liftoff force.

\subsection{Constraints}

We present here the initial conditions, constraints, and boundary conditions which must be specified in order to solve the biped walking problem. We allow a number of parameters to be variable. These are as follows:

$$
\begin{aligned}
& p_{1}=\text { step length (meters) } \\
& p_{2}=\text { magnitude of liftoff impulsive force } \\
& p_{3}=\text { time of collision (seconds) } \\
& p_{4}=\text { average forward velocity (meters } / \mathrm{min} \text { ) }
\end{aligned}
$$

At the initial and final time, periodicity of the states and controls must be enforced while in between phases continuity is enforced. If an impulsive liftoff force is included at the beginning of phase 1 , then the velocities will reflect the sudden jump caused by the impulsive force. Given the magnitude of the impulsive force $p_{2}$, the Collision Algorithm can determine the resulting new velocities for the beginning of phase 1 . The swing leg must also land at the time of collision, $p_{3}$, at a step size equal to $p_{1}$. Since in our experiments we constrain the proportion of phase 1 to $85 \%$ of the total time of the walking step, the final time may be determined from the phase 1 duration $p_{3}$.

There are additionally several constraints enforced along the duration of the walking step. We require that the vertical component of the contact forces in each phase remain positive so that there is not a premature liftoff of the leg from the ground and that the swing leg remain above ground.

\section{Minimum Energy Performance}

It is well-supported that humans follow closely an energy efficient motion strategy [9], though many issues relating consumed energy to walking remain unknown. In accomplishing our goal of generating minimal energy trajectories, we minimize the injected energy into the system. This is commonly measured as the integral of the input torques,

$$
J=\left(\int_{0}^{T_{1}} u^{T} u d t+\int_{T_{1}}^{T} u^{T} u d t\right) / \text { step length },
$$

where $T_{1}$ is the time at the end of the first phase (swing phase), and $T$ is the time at the end of the second phase (double-contact phase). Dividing by the step length, the distance between successive heel strikes, gives the expended energy per meter traveled. If an impulsive force is added, then this control parameter will also be added into the performance,

$J=\left(\int_{0}^{T_{1}} u^{T} u d t+\int_{T_{1}}^{T} u^{T} u d t+u_{i m p}^{2}\right) /$ step length.

This general form of minimal energy performance was also used in [10].

The above performance is a quantity related to the required energy for motion. For a simple actuation model, we will be minimizing the energy required for direct drive motors at the joints to produce the required torques. This approach provides a more numerically tractable way of reaching our performance objectives.

\section{Numerical Optimal Control}

Direct optimization methods for optimal control are characterized by the minimization of a cost functional which is a function of the system state and the control input $u$. An example of such a method is the program 
DIRCOL [11, 12], which can handle implicit or explicit boundary conditions, arbitrary nonlinear equality and inequality constraints on the state variables, and multiple phases where each phase may contain a different set of state equations. DIRCOL functions by packaging the optimal control problem along with its constraints into a constrained, nonlinear minimization problem which is solved by an SQP-based optimization code NPSOL [2] or SNOPT [3] which takes advantage of sparsity.

The output of the numerical optimal control program will be the optimal open-loop solution for the control $u(t)$ and the corresponding state trajectory $x(t)$ at the choice of grid points in time. DIRCOL discretizes the state and control variables in time over the trajectory. The fineness or coarseness of the discretization can have a large influence over the time required to generate a solution. The recently released DIRCOL 2.0 using sparse optimization techniques has shown itself to be faster and more robust.

As previously mentioned, there are a total of 14 states, 6 control variables, and 1 control parameter in our control problem. All of these quantities have magnitude saturation bounds placed upon them in the optimization process, though the majority never become active. The control saturations are the most important as in many experiments these will saturate, particularly at higher speeds. The bounds on the ankle torques are also smaller than those at the other joints, as the ankles cannot provide as great a force as the hips and knees can.

In addition to magnitude constraints, there are explicit constraints on the initial and final state of each phase which assign those constrained states and controls a fixed value which may depend on other known values. These are called explicit boundary conditions, while implicit boundary conditions are those for which the states and controls must satisfy a nonlinear algebraic equation. Finally, we have nonlinear inequality constraints which must be satisfied by the states and controls along the duration of the walking step. The constraints outlined in Section 2.5 result in a total of: Explicit boundary conditions: $\quad 19$ Implicit boundary conditions: $\quad 10$ Nonlinear Inequality Constraints (phase 1): 2 Nonlinear Inequality Constraints (phase 2): 3

\subsection{Optimization Trials}

The high degree of nonlinearity and high dimension of the problem, along with all the constraints, make it unreasonable to assume that by specifying the state equations, boundary conditions, and inequality constraints together with a naive initial guess of the solution, the optimization procedure will immediately find an optimal solution. Various simpler problems were first solved such as that of standing in place and then moving only small distances. In fact, an iterated process was undertaken which gradually approximated the actual problem, whereby the solution of each generalization of the problem was made using the previous one as an initialization.

For most trial runs, we used 13 grid points in time, 8 in the first phase and 5 in the second phase. As the number of grid points has a large influence on the length of each optimization run, it is preferable to use a coarse grid, then to refine the grid if more exact solutions are needed. Run times depend on the starting values given to the problem and the problem to be solved.

DIRCOL transforms the complete problem to a nonlinear optimization problem with 197 variables, 131 nonlinear equality constraints, and 23 inequality constraints. The number of function calls during a sample optimization run are:

$\begin{array}{lll}\text { DIRCOL Version } & 1.2 & 2.0 \\ \begin{array}{l}\text { Optimization Program } \\ \text { Function Calls }\end{array} & \text { NPSOL } & \text { SNOPT } \\ \quad \text { State equations } & 568635 & 230952 \\ \quad \text { Implicit Boundary Const. } & 43430 & 8497 \\ \quad \text { Nonlinear Ineq. Const. } & 249928 & 125099 \\ \text { Run Time } & 18 \mathrm{~min} . & 12 \mathrm{~min} .\end{array}$

These runs were conducted on a Sparc Ultra 2 with a $166 \mathrm{MHz}$ processor. The advantage of DIRCOL 2.0 over DIRCOL 1.2 in solving a particular problem was, in fact, much greater than the statistics above indicated. This is because several subproblems would often have to be solved with DIRCOL 1.2 before the complete problem could be solved. For example, a subproblem would be solved without enforcing positivity of the contact constraints, then the complete problem could be solved by initializing it on the solution of the subproblem. DIRCOL 2.0 would usually not require this 2stage solution process as its domain of convergence is larger, thus saving much time.

\section{Optimal Walking Experiments}

Two main categorizations can be made in that we explore first walking without any form of lift propulsion. We then add to our biped the possibility of introducing an instantaneous impulsive force at the moment of liftoff to help the body move forward. In both settings, the additional effect of using ankle torques is investigated so that we compare all together 4 distinct cases.

\subsection{Optimal Forward Velocities vs. Energy}

When energy is considered in terms of $(\mathrm{cal} / \mathrm{kg} / \mathrm{m})$, then the equation

$$
E=\frac{32}{v}+0.005 v
$$

has been shown experimentally to roughly model the relationship witnessed in humans between required energy $E$ and the average forward walking velocity $v$ [9]. This hyperbolic relationship has an energy minimizing walking velocity of $80 \mathrm{~m} / \mathrm{min}$. Figure 2 displays the relationship that we encounter in our experiments which, 


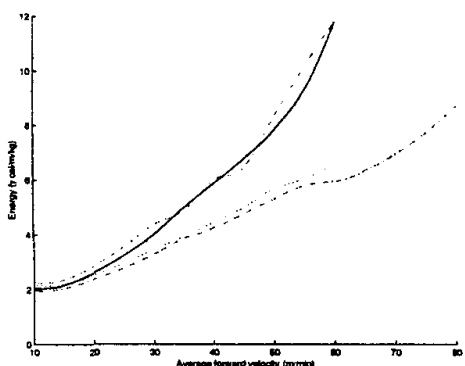

Fig 2: Required energy as a function of avg. forward velocity. With ankle torques (solid); no ankle torques (dashed); with ankle torques and liftoff force (dashdot); with liftoff force (dotted).

while reasonably hyperbolic, has a very different energy minimizing velocity of approximately $12 \mathrm{~m} / \mathrm{min}$. Corresponding to our slower globally optimal walk is also a much smaller step size of $0.1 \mathrm{~m}$ compared to $0.75 \mathrm{~m}$ witnessed in humans. A possible conjecture for the disparity with optimal human walking is the lack of the foot effect which provides essentially an extension of the leg when the back heel lifts off of the ground propelling the body forward and reducing the effects of collision.

The dashdot and dotted lines in Figure 2 indicate the energy relationship for walking with an impulsive liftoff force. A significant energy savings is obtained over walking without such a liftoff force (solid and dashed lines), though there is no noticeable change to the optimal walking speed.

\subsection{Forward Velocities and Collisions}

We display in Figure 3 the hip displacement and hip forward velocity for a complete, periodic, double step with an impulsive liftoff force. In order that the various walking trajectories may be more easily compared, we plot on the horizontal axis the normalized time for a step. The stick walking figures on the top portion of the figure indicate for the two plots beneath it which part of the walking step the plotted points correspond to. The plots begin with the swing leg leaving the ground, and ends with the same leg about to leave the ground once again.

The solid (with ankle torques) and dashed (without ankles) lines indicate optimal walking solutions. The dashdot (with ankles) and dotted (without ankles) have an additional parameter fixed which is that of the average forward velocity set at a much faster $50 \mathrm{~m} / \mathrm{min}$. The first vertical line indicates the moment of collision, the second line is the time when the second step begins with the other leg lifting off the ground, and the last line is once again a collision of the swing leg with the ground.

From the velocity plot, we see the biped loses a considerable amount of forward velocity at the moment of collision, in particular for the faster walking speed. The

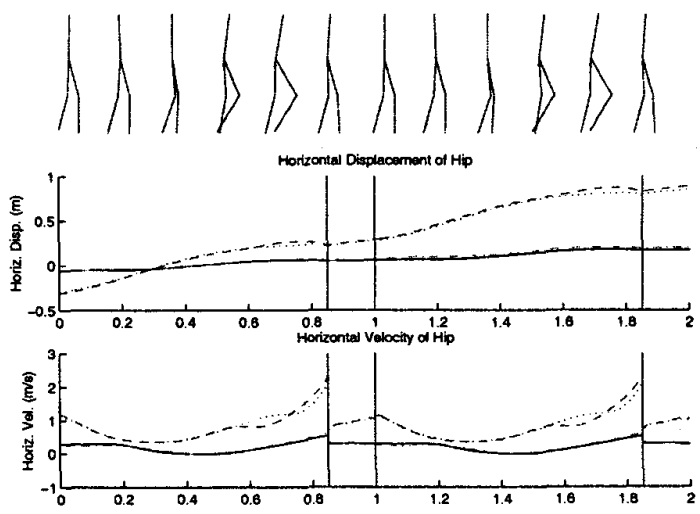

Fig 3: Optimal trajectories of hip position and velocity for walking with liftoff forces. Walk Speed: 12 $\mathrm{m} / \mathrm{min}$. No ankle torques (dashed); with ankle torques (solid). Walk Speed: $50 \mathrm{~m} / \mathrm{min}$. No ankle torques (dashed); with ankle torques (solid).

faster walking speed serves to exaggerate the overall effects as it is also more apparent that the biped needs to slow down quite a bit near the middle of the swing phase rather being able to maintain a more constant forward velocity. With the inclusion of a liftoff force, our experiments have shown that we are able to obtain more consistent forward motion and the large variation evident in the velocity plot is substantially reduced over the case when liftoff forces are not included. We speculate that without the explicit modeling of the foot, the biped cannot efficiently operate at higher speeds.

\subsection{Walking with and without Liftoff Forces}

Figures 4 and 5 display the optimal applied torques for the model with and without impulsive liftoff forces from the same set of experiments as the previous section. Including an impulsive liftoff force is an easy way of modeling the same effects as having a foot. The solid and dashed lines indicate the torques for the optimal walking motion for the model with and without ankle actuation respectively. There is a notable difference between the $12 \mathrm{~m} / \mathrm{min}$ (globally optimal) and the $50 \mathrm{~m} / \mathrm{min}$ walk. It is evident that the torques for the faster walk reach the magnitude constraints placed upon them several times. The torque saturations can be greatly reduced with the introduction of an impulsive liftoff force. These torques (and their rates) are of much smaller magnitude showing the beneficial effect this additional control parameter has.

For a given velocity, it is difficult to distinguish the difference between including and not including ankle actuation. The difference only becomes visible at the higher forward velocity when during the second phase a slightly smoother and smaller torque actuation is required for the hip and knee. An interesting effect witnessed in our experiments is that the knee comes more into use with ankle actuation. As a result, the torque 

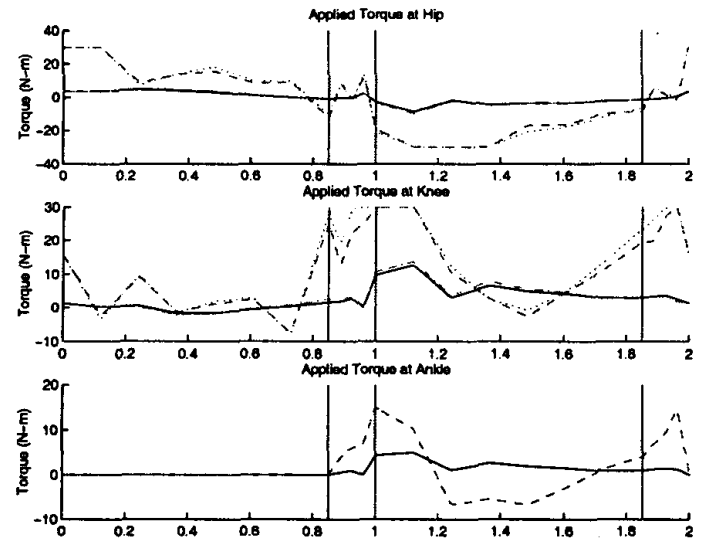

Fig 4: Optimal applied torques for walking without liftoff forces. Walk Speed: $12 \mathrm{~m} / \mathrm{min}$. No ankle torques (dashed); with ankle torques (solid). Walk Speed: $50 \mathrm{~m} / \mathrm{min}$. No ankle torques (dashed); with ankle torques (solid).

inputs and associated cost in injected energy will be more equally distributed through the different joints of the biped.

\section{Conclusion}

Our investigation into the generation of minimum energy symmetric, periodic gaits gathers together several different research areas in the modeling and control of complex, nonlinear systems. Our ability to solve this problem has relied upon the use of recursive, symbolic multibody algorithms coupled with powerful numerical optimal control software. Some of the more interesting conclusions that can be made from our experiments are:

- Beneficial use of impulsive liftoff forces to prevent torque saturation, to smooth the walking motion, and to reduce the required energy.

- Biped model without explicit modeling of feet has a much slower walk with shorter steps than optimal human walk.

- Ankle actuation smoothens the walking step and distributes the required torque input from the hip to the knee and ankle.

\section{Acknowledgements}

We would like to express our gratitude to Oskar von Stryk for making his program available to us. Also, we are indebted to Oskar von Stryk and Michael Breitner for their helpful comments regarding this problem and the use of this numerical, optimization approach.

\section{References}

[1] C.K. Chow and D.H. Jacobson, "Studies of Human Locomotion via Optimal Programming," Math. Biosciences, 10, pp. 239-306, 1971.
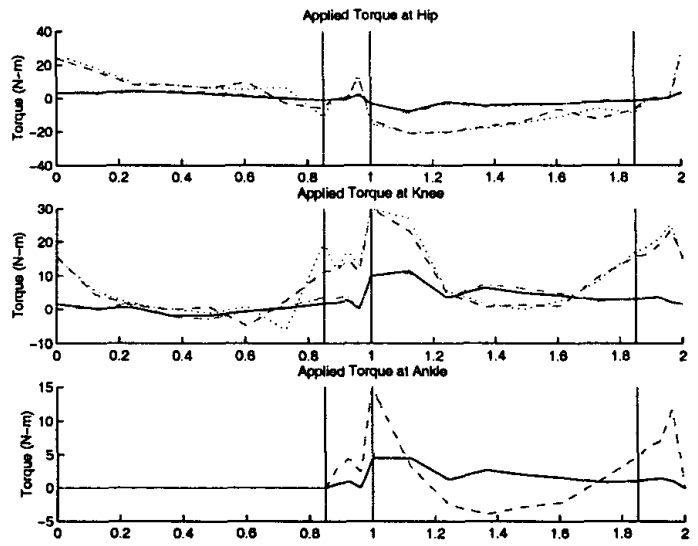

Fig 5: Optimal applied torques for walking with liftoff forces. Walk Speed: $12 \mathrm{~m} / \mathrm{min}$. No ankle torques (dashed); with ankle torques (solid). Walk Speed: $50 \mathrm{~m} / \mathrm{min}$. No ankle torques (dashed); with ankle torques (solid).

[2] P.E. Gill, W. Murray, M.A. Saunders, M.H. Wright, User's Guide for NPSOL 5.0.

[3] P.E. Gill, W. Murray, and M.A. Saunders, SNOPT: An SQP algorithm for large-scale constrained optimization, Num. Anal. Rep. 97-2, Dept. of Math., UCSD, La Jolla, CA, 1997.

[4] A. Goswami, B. Espiau, and A. Keramane, "Limit Cycles in a Passive Compass Gait Biped and Passivity-Mimicking Control Laws," Autonomous Robots, 4, pp. 273-286, 1997.

[5] M. Hardt, Multibody Dynamical Algorithms, $\mathrm{Nu}$ merical Optimal Control, with Detailed Studies in the Control of Jet Engine Compressors and Biped Walking, Ph.D. Thesis, Elec. Eng., UCSD, La Jolla, CA, 1999.

[6] M. Hardt, K. Kreutz-Delgado, and J. William Helton, 37th IEEE CDC, pp. 413-6, 1998.

[7] S. Kajita and K. Tani, "Experimental Study of Biped Dynamic Walking," IEEE Control Systems, pp. 13-19, 1996.

[8] T. McGeer, "Passive Dynamic Walking," The Int'l J. of Rob. Res. 9(2), pp. 62-82, Apr 1990.

[9] J. Rose and J.G. Grimble, Human Walking, Williams \& Wilkins, 1994.

[10] L. Roussel, C. Canudas de Wit, and A. Goswami, "Generation of Energy Optimal Complete Gait Cycles for Biped Robots," Proc. of the 1998 IEEE Rob. E Aut., pp. 2036-41.

[11] O. von Stryk, User's Guide for DIRCOL 2.0. A direct collocation method for the numerical solution of optimal control problems, 1999.

[12] O. von Stryk, In: R. Bulirsch, A. Miele, J. Stoer, K.-H. Well (eds.): Optimal Control, Int'l Ser. in Numerical Math. 111, Birkhäuser, pp. 129-143, 1993. 